\title{
ORIGINAL
}

\section{PREVALENCIA, DETECCIÓN, TRATAMIENTO Y CONTROL DE LA HIPERTENSIÓN ARTERIAL EN CANTABRIA EN 2002 (*)}

\author{
Luis Vara-González (1), Pedro Muñoz Cacho (2), Saturnino Sanz de Castro (3) \\ Hospital Universitario Marqués de Valdecilla. \\ (1) Centro de Salud La Marina. Santander (Cantabria). \\ (2) Gerencia de Atención Primaria Santander-Laredo. \\ (3) Servicio de Nefrología. Hospital Marqués de Valdecilla.
}

En representación de los investigadores del grupo CANHTABRIA: Angélica Saiz Berzosa, Eulalia Aguilera Zubizarreta, María Casaus Pérez, Mercedes Castaño García, Ana Cuesta Pérez-Camino, Cristina Díez Cruz, Daniel García Barrigón, Mª . José García Zarrabeitia, Rosa González Fernández, J. Ramón López Lanza, Patricia Martínez Fernández, Graciela Saiz Fernández, Vanessa Sierra de Pablo, Teresa Ugarte Miota.

* Este estudio ha sido financiado con becas de MSD-España, del Gobierno de Cantabria (10.4.412A.482/2003) y de la Fundación Marqués de Valdecilla (API 04/13).

\section{RESUMEN}

Fundamento: La hipertensión arterial es uno de los principales factores de riesgo cardiovascular. El objetivo de este estudio fue conocer en Cantabria la prevalencia de la hipertensión arterial, así como los grados de detección, tratamiento y control.

Métodos: Entre 2002 y 2004 se realizaron mediciones de presión arterial y se recogieron otros datos generales en las personas de una muestra aleatoria de 1.197 individuos de 18 o más años de edad, elegida en varias etapas y estratificada por edad y sexo.

Resultados: La prevalencia de hipertensión encontrada fue del $29 \%$, siendo similar en hombres (29\%) y en mujeres $(28 \%)$. Entre las personas hipertensas el $65 \%$ conocía su situación, el $53 \%$ recibía tratamiento y el $22 \%$ presentaba cifras por debajo de $140 / 90 \mathrm{~mm} \mathrm{Hg}$.

Conclusiones: La hipertensión arterial afecta a 1 de cada 3 sujetos adultos de la población cántabra. Aunque los niveles de detección, tratamiento y control son superiores a los descritos en esta Comunidad Autónoma en 1989, la situación es claramente mejorable.

Palabras clave: Hipertensión arterial. Estudios transversales. Epidemiología. Prevalencia. Presión arterial. Detección. Tratamiento. Control. Antihipertensivos. Cantabria. España.

Correspondencia:

Luis Vara-González

C/ Dr. Diego Madrazo 10, 4º B. 39012 Santander

Correo electrónico: luvara@ono.com

\section{ABSTRACT}

\section{Arterial Hypertension Prevalence, Detection, Treatment and Control in Cantabria, Spain, 2002}

Background: Hypertension is a major cardiovascular risk factor. The aim of this study was to establish the prevalence of high blood pressure in a European region in Northern Spain (Cantabria), and also levels of detection, treatment, and control.

Methods: On a random multi-stage sample of 1197 individuals aged 18 or over, stratified by sex and age, blood pressure measurements were taken and other general data were collected from 2002 to 2004.

Results: A prevalence of hypertension of $29 \%$ was found. The prevalence among males and females was similar, being $29 \%$ and $28 \%$, respectively. Sixty five per cent of the hypertensive subjects were aware of their condition, $53 \%$ were undergoing treatment, and $22 \%$ presented readings below $140 / 90 \mathrm{~mm} \mathrm{Hg}$.

Conclusions: Hypertension constitutes a public health problem in Cantabria, since it affects 1 in 3 of the adult population. Although levels of detection, treatment, and control are superior to those previously described in this region in 1989 , the situation could clearly be improved.

Key words: Hypertension. Cross-sectional studies. Epidemiology. Prevalence. Blood pressure. Detection. Treatment. Control. Antihypertensive drugs. Cantabria. Spain. 


\section{INTRODUCCIÓN}

La cardiopatía isquémica y el ictus son la primera y segunda causas de muerte en el mundo ${ }^{1}$. Su asociación con la hipertensión arterial (HTA) hace que ésta sea el factor de riesgo con una mayor mortalidad atribuible $^{2}$. La prevalencia de HTA encontrada en las diferentes regiones del mundo es muy variable, así en la revisión realizada por Kearney ${ }^{3}$ en 2004 se describe una prevalencia que varía desde la encontrada en la India rural (3\% en los varones y $7 \%$ en las mujeres) a la registrada en Polonia (69\% en los varones y $73 \%$ en las mujeres). Algunas estimaciones realizadas en el año 2000 situaban la prevalencia de la HTA en el $26 \%$ de la población adulta mundial $^{4}$. Asimismo, se considera que dentro del área de países desarrollados Europa presenta una mayor prevalencia $(44 \%)$ que Norteamérica $(28 \%)^{5}$. En España se han publicado numerosos estudios sobre la prevalencia de la HTA de ámbito local o regional en la población general, pero existen muy pocos de carácter nacional. Entre ellos cabe destacar los realizados por Banegas et al en 1990 y 2001. En el primero ${ }^{6}$, realizado en sujetos de 35 a 64 años de edad, se encontró una prevalencia de HTA del $45 \%$, y en el segundo ${ }^{7}$, realizado en sujetos de 60 o más años de edad, del $68 \%$. Si variable es la prevalencia detectada de la HTA en las diversas poblaciones estudiadas también lo son los grados de detección, tratamiento y control. De tal modo que en la mencionada revisión de Kearney ${ }^{3}$ la detección variaba desde el 25 al $75 \%$, el tratamiento desde el 11 al $66 \%$ de los individuos hipertensos y el grado de control desde el 5 al 58\%. Aunque los grados de detección, tratamiento y control han mejorado en algunos países, se considera que estos siguen siendo inadecuados ${ }^{3}$. En España algunas estimaciones ${ }^{8}$ situaban la detección en el $65 \%$, el tratamiento en el $55 \%$ y el control en el $15 \%$, en el año 2002. Las estimaciones fiables acerca de su prevalencia y control, y su evolución en el tiempo, son esenciales para la planificación de los servicios sanitarios 9 .

El objetivo de este estudio fue conocer la situación epidemiológica de la hipertensión arterial en Cantabria.

\section{SUJETOS Y MÉTODOS}

Tipo de estudio: Se realizó un estudio transversal en una muestra representativa de la población general adulta de Cantabria, formada por 1.197 individuos de $18 \mathrm{o}$ más años de edad. Cantabria es una región situada en el norte de España que en 2002 contaba con una población de 542.275 habitantes. Se utilizó un muestreo poli-etápico, aleatorio, estratificado por edad y sexo. En primer lugar se eligieron varios municipios y posteriormente varios centros de salud y cupos médicos. Del listado de cada cupo médico elegido se seleccionó a los individuos de la muestra de manera estratificada por edad y sexo. Se utilizó el listado de la tarjeta individual sanitaria del Servicio Cántabro de Salud, cuya cobertura está próxima al $100 \%$ de la población total de Cantabria. El tamaño de la muestra se calculó para una prevalencia estimada ${ }^{5}$ del $40 \%$, un nivel de confianza del $95 \%$ y una precisión en la estimación de $\pm 4 \%$. En total, los individuos pertenecían a 31 cupos médicos (de los 305 que había en ese momento en Cantabria) de 21 municipios (de un total de 102). Tras informar a los individuos seleccionados mediante una carta con los objetivos del estudio, se concertó una cita en su domicilio o en su consultorio correspondiente si el sujeto así lo prefería. Los individuos que no participaron en el estudio fueron sustituidos por otros, elegidos previamente de manera aleatoria, de la misma edad, sexo y cupo médico. En total se realizaron 504 sustituciones: el 18\% de los individuos rechazó participar en el estudio (no respondedores), el 17\% no pudo ser localizado y el $7 \%$ había fallecido antes del inicio del estudio o vivía fuera de Can- 
tabria durante la realización del mismo. Mediante llamada telefónica realizada por los entrevistadores se obtuvieron datos sobre consumo de tabaco, peso, talla, nivel educativo y presencia de HTA en el $66 \%$ de los casos de los sujetos que rechazaron participar en el estudio. El conjunto de sujetos no respondedores presentó un porcentaje mayor de fumadores ( $42 \%$ vs $34 \%$ en los respondedores, $\mathrm{P}<0,05)$, su índice de masa corporal era menor $\left(25 \mathrm{~kg} / \mathrm{m}^{2}\right.$ vs $27 \mathrm{~kg} / \mathrm{m}^{2}$ en los respondedores, $\left.\mathrm{P}<0,01\right)$ y un nivel educativo medio-alto menor $(28 \%$ vs $49 \%$ en los respondedores, $\mathrm{P}<0,001)$. No hubo diferencias en cuanto al sexo, edad ni diagnóstico de HTA. El estudio fue aprobado por el Comité ético de investigación del Hospital Universitario Marqués de Valdecilla y se realizó con el consentimiento informado de todas las personas participantes.

Mediciones: Los datos fueron recogidos por 14 investigadores especialmente entrenados y acreditados para realizar la entrevista y las exploraciones, desde octubre de 2002 a diciembre de 2004. Se recogieron datos sobre consumo de tabaco, de bebidas alcohólicas, presencia de enfermedades cardiovasculares, HTA u otros factores de riesgo cardiovascular, y acerca del tratamiento. Después de cumplimentar el cuestionario se realizaron mediciones de peso, talla, frecuencia cardiaca y presión arterial (PA): 6 en sedestación, 3 con esfigmomanómetro de mercurio estándar e intercaladas 3 con un dispositivo semiautomático OMRON $705 \mathrm{CP}$ y 2 en bipedestación tras uno y tres minutos. El índice de masa corporal (IMC) se calculó dividiendo el peso $(\mathrm{kg})$ por el cuadrado de la talla $\left(\mathrm{m}^{2}\right)$. Todas las mediciones se realizaron de manera estandarizada y según recomiendan las guías de práctica clínica y las sociedades científicas, tras 5 minutos de reposo, evitando en la hora previa el consumo de café, alcohol, tabaco o cualquier alimento, la exposición al frío y la práctica de ejercicio ${ }^{10,11}$. Se ha utilizado la media de las dos últimas mediciones realizadas en sedestación con el esfigmomanómetro de mercurio o de las tres en los individuos con arritmia. Durante todo el estudio se realizó un control continuado de la calidad de las mediciones realizadas y de la correcta calibración de los aparatos.

\section{Definiciones utilizadas en el estudio:}

Se consideró hipertenso a todo individuo que presentase una PA igual o superior a 140/90 mm Hg o que estuviese en tratamiento con fármacos antihipertensivos para tratar un problema de hipertensión arterial. De ellos los que declararon haber sido diagnosticados de HTA fueron considerados casos conocidos. Fueron considerados hipertensos en tratamiento aquellos individuos que recibían tratamiento con fármacos antihipertensivos y controlados aquellos individuos cuya cifra de presión arterial era $<140 / 90 \mathrm{~mm} \mathrm{Hg}$.

Análisis estadístico: Se realizaron las 8 mediciones en 1.174 sujetos (98\%). Las causas principales de la falta de alguna medición fueron la negativa del sujeto, la falta de lectura del aparato electrónico o la imposibilidad para mantenerse de pie el tiempo suficiente. Se utilizó el programa SPSS versión 11.0 para Windows. Los resultados de las variables cualitativas se han expresado en porcentajes con sus correspondientes intervalos de confianza del 95\% (IC95\%) y los de las variables cuantitativas con la media, su desviación estándar e IC95\%. Con el propósito de facilitar la comparación de los resultados con otros estudios se ha calculado la presión arterial media y la prevalencia de HTA ajustadas para la población mundial estándar de $2002^{12}$. Para los contrastes de hipótesis que se relacionan con las variables cualitativas se ha utilizado la prueba estadística de $\mathrm{chi}^{2}$, para las variables cuantitativas el test de la t de Student. Se ha realizado un análisis de regresión logística para comprobar la asociación entre la presencia de HTA y el resto de variables estudiadas. Se 
Tabla 1

Presión sistólica y diastólica media en la población

\begin{tabular}{|c|c|c|c|}
\hline $\begin{array}{c}\text { Presión } \\
\text { sistólica }\end{array}$ & $\begin{array}{c}\text { IC } \\
\text { IC 95\% }\end{array}$ & $\begin{array}{c}\text { Presión } \\
\text { diastólica }\end{array}$ & $\begin{array}{c}\text { IC } \\
\text { IC 95\% }\end{array}$ \\
\hline 123,9 & $122,8-125,1$ & 76,1 & $75,5-76,7$ \\
\hline
\end{tabular}

han considerado estadísticamente significativos los valores de $\mathrm{p}<0,05$.

\section{RESULTADOS}

\section{Características de los participantes:} La media de edad fue de 49 años (DE: $18,8)$, con una mediana de 46 y un rango entre 18 y 100 años. El 54\% eran mujeres. Fumaba el $34 \%$ de los sujetos incluidos y tenía un consumo excesivo de bebidas alcohólicas el 10\%. El 22\% de los sujetos eran obesos y el $37 \%$ presentaban sobrepeso. Respecto a las otras variables estudiadas: el $8 \%$ declaró padecer diabetes mellitus, el $23 \%$ hiperlipidemia, el $8 \%$ una cardiopatía y el $3 \%$ una enfermedad cerebrovascular.

Presión arterial: Las PA medias obtenidas se recogen en la tabla 1 . La PAS media en los varones fue superior a la encontrada en las mujeres (126 vs $122 \mathrm{~mm}$ $\mathrm{Hg}$, respectivamente, $P<0,001)$ y también la PAD (78 vs $74 \mathrm{~mm} \mathrm{Hg}$, respectivamente, $P<0,001)$ sin embargo, la presión de pulso media fue similar en ambos sexos (48 mm Hg). La PA fue mayor en los varones hasta los 69 años y en las mujeres la PAS a partir de los 80 años de edad (tabla 2). La PA estaba asociada a la edad y al índice de masa corporal $(P<0,001$, para ambas). La PA media ajustada por la edad para la población mundial estándar fue de 119/74 mm Hg.

Prevalencia de HTA: La prevalencia de HTA fue del $28,7 \%$, aumentaba con la edad y con el índice de masa corporal (tabla 3). El efecto de la edad y del IMC, ajustado por las otras variables, fue estadísticamente significativo $(P<0,001$, en ambos). La prevalencia estandarizada según la población mundial fue del 19,5\%. Sólo el $42 \%$ de los individuos presentaba unas cifras de PA óptimas, según las categorías $^{11}$ del JNC 7.

\section{Conocimiento, tratamiento y control:} De los 343 hipertensos, el 65\% sabía que lo era (tabla 4). Entre ellos el $81 \%$ se encontraba en tratamiento con fármacos antihipertensivos (53\% de todos los hipertensos), y, de los tratados, presentaban un buen control de sus cifras de PA el $41 \%$ (22\% de todos los hipertensos). La detec-

Tabla 2

Presión arterial media sistólica y diastólica $(\mathrm{mm} \mathrm{Hg}$ ) según la edad y el sexo

\begin{tabular}{|c|c|c|c|c|c|c|c|c|}
\hline \multirow{3}{*}{$\begin{array}{c}\text { Grupo de } \\
\text { edad (años) }\end{array}$} & \multicolumn{4}{|c|}{ Presión arterial sistólica } & \multicolumn{4}{|c|}{ Presión arterial diastólica } \\
\hline & \multicolumn{2}{|c|}{ Varones } & \multicolumn{2}{|c|}{ Mujeres } & \multicolumn{2}{|c|}{ Varones } & \multicolumn{2}{|c|}{ Mujeres } \\
\hline & Media & IC $95 \%$ & Media & IC 95\% & Media & IC $95 \%$ & Media & IC $95 \%$ \\
\hline $18-29$ & $119 *$ & $(117-121)$ & 107 & (106-109) & $73 *$ & $(72-75)$ & 67 & $(66-69)$ \\
\hline $30-39$ & $120 *$ & $(118-122)$ & 108 & $(106-111)$ & $76 *$ & $(75-78)$ & 70 & $(68-71)$ \\
\hline $40-49$ & $125^{*}$ & $(122-128)$ & 113 & $(110-116)$ & $82 *$ & $(80-83)$ & 74 & $(72-75)$ \\
\hline $50-59$ & $129^{* *}$ & $(126-133)$ & 124 & $(120-127)$ & $82^{* * *}$ & $(80-84)$ & 78 & $(76-80)$ \\
\hline $60-69$ & $139^{* * *}$ & $(135-144)$ & 133 & $(128-137)$ & $83^{* *}$ & $(80-85)$ & 79 & $(77-81)$ \\
\hline $70-79$ & 140 & $(133-147)$ & 145 & $(140-149)$ & 79 & $(77-82)$ & 80 & $(78-83)$ \\
\hline$\geq 80$ & $131^{* * *}$ & $(121-141)$ & 145 & $(138-152)$ & 73 & $(67-78)$ & 76 & (73-79) \\
\hline
\end{tabular}

$*: \mathrm{P}<0,001 . * *: \mathrm{P}<0,05$. Entre varones y mujeres. 
Tabla 3

Prevalencia de hipertensión, con el intervalo de confianza (IC) correspondiente y número de hipertensos en los diferentes grupos de edad, sexo e índice de masa corporal

\begin{tabular}{|c|c|c|c|c|c|c|c|c|c|}
\hline \multicolumn{10}{|c|}{ Hipertensión arterial } \\
\hline & \multicolumn{3}{|c|}{ Todos } & \multicolumn{3}{|c|}{ Varones } & \multicolumn{3}{|c|}{ Mujeres } \\
\hline & $\mathbf{N}$ & $\%$ & IC $95 \%$ & $\mathbf{N}$ & $\%$ & IC $95 \%$ & $\mathbf{N}$ & $\%$ & IC $95 \%$ \\
\hline Todos & 343 & 28,7 & $26,1-31,3$ & 161 & 29,4 & $25,6-33,4$ & 182 & 28,0 & $24,7-31,7$ \\
\hline \multicolumn{10}{|c|}{ Edad, en años } \\
\hline $18-39$ & 20 & $4,4^{*}$ & $2,8-6,9$ & 15 & $6,5^{*}$ & $3,8-10,7$ & 5 & $2,3^{*}$ & $0,8-5,5$ \\
\hline $40-59$ & 104 & 25,9 & $21,8-30,6$ & 67 & 35,3 & $28,6-42,6$ & 37 & 17,5 & $12,8-23,5$ \\
\hline$\geq 60$ & 219 & $63,7^{*}$ & $58,3-68,7$ & 79 & $61,7^{*}$ & $52,7-70,1$ & 140 & $64,8^{*}$ & $58,0-71,1$ \\
\hline \multicolumn{10}{|c|}{ IMC $\left(\mathrm{kg} / \mathrm{m}^{2}\right)$} \\
\hline$<25$ & 53 & 11,1 & $8,5-14,4$ & 20 & 11,2 & $7,1-16,9$ & 33 & 11,1 & $7,9-15,3$ \\
\hline $25-29,9$ & 151 & $33,8^{*}$ & $29,4-38,4$ & 73 & $30,7^{*}$ & $25,0-37,0$ & 78 & $37,3^{*}$ & $30,8-44,3$ \\
\hline$\geq 30$ & 137 & $51,7^{*}$ & $45,5-57,8$ & 66 & $52,4^{*}$ & $43,3-61,3$ & 71 & $51,1^{*}$ & $42,5-59,6$ \\
\hline
\end{tabular}

*: p $<0,001$. Las categorías de referencia son la edad, entre 40-59 años, y el normopeso (IMC < 25). IMC: Índice de masa corporal.

Tabla 4

Detección, tratamiento y control de la hipertensión arterial en Cantabria según edad, sexo e índice de masa corporal

\begin{tabular}{|c|c|c|c|c|c|c|}
\hline & \multicolumn{2}{|c|}{ Detección } & \multicolumn{2}{|c|}{ Tratamiento en detectados } & \multicolumn{2}{|c|}{ Control en tratados } \\
\hline & $\%$ & IC $95 \%$ & $\%$ & IC $95 \%$ & $\%$ & IC $95 \%$ \\
\hline Todos & 65,3 & $59,9-70,3$ & 80,8 & $74,9-85,6$ & 41,4 & $34,2-49,0$ \\
\hline \multicolumn{7}{|l|}{ Sexo } \\
\hline Varones & 55,9 & $47,9-63,6$ & 74,4 & $64,0-82,8$ & 55,2 & $42,6-67,2$ \\
\hline Mujeres & $73,6^{\mathrm{a}}$ & $66,5-79,7$ & $85,1^{\mathrm{b}}$ & $77,7-90,4$ & $33,3^{\mathrm{a}}$ & $25,0-42,9$ \\
\hline \multicolumn{7}{|c|}{ Edad, en años } \\
\hline $18-39$ & 45,0 & $23,8-68,0$ & $22,2^{\mathrm{a}}$ & $4,0-59,8$ & 50,0 & $2,7-97,3$ \\
\hline $40-59$ & 52,0 & $42,0-61,7$ & 72,2 & $58,1-83,1$ & 59,0 & $42,2-74,0$ \\
\hline$\geq 60$ & $73,5^{\mathrm{c}}$ & $67,1-79,1$ & $87,0^{\mathrm{b}}$ & $80,5-91,6$ & $36,4^{b}$ & $28,6-45,0$ \\
\hline \multicolumn{7}{|c|}{$\mathrm{IMC}\left(\mathrm{kg} / \mathrm{m}^{2}\right)$} \\
\hline$<25$ & 54,7 & $40,6-68,2$ & 82,8 & $63,5-93,5$ & 25,0 & $10,6-47,1$ \\
\hline $25-29,9$ & 65,6 & $57,3-73,0$ & 83,8 & $74,8-90,2$ & 47,0 & $36,1-58,2$ \\
\hline$\geq 30$ & 69,3 & $60,8-76,8$ & 76,8 & $66,8-84,6$ & 39,7 & $28,7-51,9$ \\
\hline
\end{tabular}

a: $\mathrm{P}<0,01$. b: $\mathrm{P}<0,05$. c: $\mathrm{P}<0,001$. Las categorías de referencia son: el sexo masculino, la edad, entre 40-59 años, y el normopeso (índice de masa corporal $<25 \mathrm{~kg} / \mathrm{m}^{2}$ ). IMC: índice de masa corporal.

ción y el grado de tratamiento fueron superiores en las mujeres y en los individuos de más edad. Sin embargo, el grado de control de la PA entre los sujetos que recibían tra- tamiento fue mayor en los varones y en los sujetos más jóvenes. Entre todos los hipertensos el control de la PAD fue superior (64\%) que el de la PAS (33\%). 
Tipo de tratamiento: $\mathrm{Si}$ se incluye a los sujetos que declararon haber sido diagnosticados de HTA y cuyas cifras de PA encontradas en el estudio fueron normales la prevalencia de HTA sería del $32 \%$. Estos individuos podrían haber conseguido normalizar sus cifras de PA con medidas del estilo de vida exclusivamente. De los hipertensos en tratamiento con antihipertensivos, el 54\% estaba en monoterapia, el $38 \%$ tomaba dos antihipertensivos, y el resto tres o más fármacos. El grupo más utilizado fue el de los diuréticos (36\%) seguido por los inhibidores de la enzima conversora de la angiotensina (23\%). La combinación más empleada fue la que asociaba un diurético con un inhibidor de la enzima conversora de la angiotensina (34\%). Los diuréticos fueron los fármacos más utilizados en combinación, ya que estaban presentes en el $80 \%$ de los casos.

\section{DISCUSIÓN}

La HTA es un problema de alta prevalencia en Cantabria, ya que afecta a uno de cada tres adultos y su situación es claramente mejorable ya que todavía una de cada tres personas hipertensas no está diagnosticada, una de cada dos no recibe tratamiento antihipertensivo y sólo una de cada cinco tiene controladas sus cifras de presión arterial. La detección es menor en los varones jóvenes. La obesidad es un importante factor predictor de la HTA ya que el $52 \%$ de los individuos obesos la padecen mientras que sólo se presenta en el 11\% de los sujetos con peso normal.

Creemos que los datos obtenidos en este estudio son fiables ya que las mediciones de PA se realizaron de la manera estandarizada que recomiendan las guías de práctica clínica y fueron realizadas por entrevistadores especialmente entrenados y acreditados con instrumentos bien calibrados y validados para la práctica clínica ${ }^{13}$. Además, se realizó un control continuo de la calidad de las mediciones ${ }^{14}$ que permitió constatar que ésta fue alta a lo largo de todo el estudio. El índice de respuesta obtenido y la distribución de los individuos de la muestra, similar a la de la población general de Cantabria, hacen que los datos sean aplicables a dicha población. Para corregir las desviaciones que podría originar la falta de respuesta de algunos individuos se han obtenido algunos datos que pueden identificar rasgos diferentes de los individuos no respondedores que pudieran estar relacionados con la presencia de la HTA. La única variable diferente de los individuos no respondedores asociada con la presencia de HTA fue el IMC, que fue superior en los individuos respondedores, aunque esta diferencia podría explicarse por el método empleado en la obtención de los datos, que en el caso de los no respondedores fue su propia declaración, lo que puede conllevar una infraestimación del peso. En todo caso, la prevalencia de HTA estimada, al considerar el menor IMC de los sujetos no respondedores, sería del $27,9 \%$.

Este estudio tiene también algunas limitaciones que deben ser señaladas: el porcentaje de individuos que no pudieron ser localizados es más alto que en otros estu$\operatorname{dios}^{7}$. Esto se debió a los numerosos cambios de compañía telefónica que se han producido en Cantabria por el inicio de la actividad de las compañías de telefonía por cable. En principio, no parece que esta circunstancia esté relacionada con ninguna variable incluida en el estudio. En el presente estudio no se han realizado mediciones repetidas en los mismos individuos en días y horas y diferentes, lo que hubiera proporcionado una mayor aproximación a la PA habitual de cada individuo?

La prevalencia de la HTA en Cantabria, en relación a la descrita en estudios realizados en otras regiones, provincias o ciudades españolas, en los que se ha incluido una población adulta, se sitúa en un lugar intermedio, similar a la descrita en Navarra ${ }^{15}$, Mur- 
cia $^{16}$, Andalucía ${ }^{17}$, Comunidad Valenciana $^{18}$ y Albacete ${ }^{19}$, en torno al $30 \%$; superior a la descrita en Huelva ${ }^{20}$, Burgos ${ }^{21}$ y Alicante $^{22}$, en torno al $25 \%$, e inferior a la encontrada en Orense $\mathrm{e}^{23}, 35 \%$.

Un reciente meta-análisis ${ }^{24}$ de estudios realizados en diferentes lugares de España sitúa la prevalencia de la HTA en el $34 \%$, lo que coincide con las estimaciones recogidas en la Guía sobre el diagnóstico y tratamiento de la HTA en España $2005^{8}$.

La prevalencia de HTA encontrada en Cantabria en sujetos de 60 o más años de edad es del $64 \%$, similar a la descrita en el estudio nacional ${ }^{7}$ realizado en este grupo de edad, que fue del $68 \%$. En el grupo de individuos de 35-64 años de edad la prevalencia encontrada en Cantabria es inferior, $25 \%$, a la descrita en el estudio nacional ${ }^{6}$ realizado en este grupo de edad, en el que fue del $45 \%$.

En relación a otros países desarrollados, la prevalencia de HTA en Cantabria es sólo ligeramente superior a la descrita en EE $\mathrm{UU}^{25}$ en el 1999-2000 NHANES, que fue del $27,1 \%$. En el estudio americano la media de edad de los participantes fue de 43 años en los varones y 45 años en la mujeres, mientras que en el estudio de Cantabria, la fue de 49 y 51, respectivamente. Dada la asociación existente entre la edad y la presencia de la HTA, esta diferencia de prevalencia puede ser explicada por las diferencias de edad. De hecho, al analizar la prevalencia por grupos de edad, las cifras son algo inferiores en Cantabria: 7,8 y 4,4\% (18-30 años); 30,6 y 25,9\% (40-59 años), y 64,5 y $63,7 \%$ (en mayores de 59 años). Si comparamos la prevalencia ajustada por la edad según la población mundial estándar en Cantabria fue de un 19,5\%, ligeramente inferior a la de EEUU: 20,3\% y Canadá: $21,4 \%{ }^{3}$. La prevalencia estimada a nivel mundial $^{4}$ se situaría en torno al $26 \%$.

Aunque los grados de detección (65\%), tratamiento $(53 \%)$ y control $(22 \%)$ son mejores que los descritos en Cantabria hace 17 años ${ }^{26}$ : detección del $45 \%$, tratamiento del $22 \%$ y control del $5 \%$, son inferiores a los descritos en $\mathrm{EE} \mathrm{UU}^{25}$ : detección del $69 \%$, tratamiento del $58 \%$ y control del $31 \%$, y se sitúan dentro de los valores descritos en la región mundial de países desarrollados ${ }^{3}$. Con respecto a las estimaciones realizadas en España ${ }^{8}$, la situación en Cantabria se asemejaría en los niveles de detección y tratamiento, que se sitúan en el $65 \%$ y $55 \%$, respectivamente, y sería algo superior en el grado de control, que se sitúa en el $15 \%$.

En concordancia con otros estudios la obesidad se asocia a la presencia de HTA pero sin embargo no hubo una mayor detección ni tratamiento en estos individuos ${ }^{7}$.

Esta mejoría en la situación epidemiológica de la HTA podría estar relacionada con la reforma sanitaria realizada en España en los últimos años donde la cobertura sanitaria pública está próxima al $100 \%$. A pesar de que la situación ha mejorado, nos encontramos muy lejos de los niveles óptimos, como por ejemplo, los propuestos por el US Departement of Health and Human Services en su informe para el año 2010 que recomienda la disminución de la prevalencia de HTA a una $16 \%$, elevar el tratamiento a un $95 \%$ y el grado de control al $50 \%$ de todos los hipertensos ${ }^{25}$. También dentro de las estrategias del Sistema Nacional de Salud español se encuentra como objetivo prioritario el control de la $\mathrm{HTA}^{27}$. Para intentar alcanzar estos objetivos sería necesario poner en marcha actividades en el ámbito poblacional e individual y desarrollar exámenes periódicos de salud para confirmar el logro real de los objetivos propuestos. La modificación en el estilo de vida de los sujetos con prehipertensión podría formar parte de esta intervención para prevenir la aparición de la HTA ${ }^{11}$.

En conclusión, la HTA tiene una elevada prevalencia en Cantabria y constituye 
un problema de salud pública de primer orden. Aunque la detección, tratamiento y control han mejorado en los últimos años, estamos muy lejos de los niveles adecuados, por lo que se debería poner en marcha alguna actividad encaminada a mejorar esta situación de manera prioritaria.

\section{AGRADECIMIENTOS}

Los autores quieren expresar su agradecimiento al Profesor José Ramón Banegas por su colaboración en el diseño de este proyecto.

\section{BIBLIOGRAFÍA}

1. Mathers CD, Loncar D. Projections of Global Mortality and Burden of Disease from 2002 to 2030. PLoS Med. 2006; 3: 2011-2030.

2. Ezzati M, Lopez AD, Rodgers A, Vander Hoorn S, Murray CJ, and the Comparative Risk Assessment Collaborating Group. Selected major risk factors and global and regional burden of disease. Lancet. 2002; 360: 1347-1360.

3. Kearney PM, Whelton M, Reynolds K, Whelton PK, He J. Worldwide prevalence of hypertension: a systematic review. J Hypertens. 2004; 22: 1119.

4. Kearney PM, Whelton M, Reynolds K, Muntner P, Whelton PK, He J. Global burden of hypertension: analysis of worldwide data. Lancet. 2005; 365: 217-223.

5. Wolf-Maier K, Cooper RS, Banegas JR, Giampaoli S, Hense H-W, Joffres M, et al. Hypertension prevalence and blood pressure levels in 6 European countries, Canada, and the United States. JAMA. 2003; 289: 2363-2369.

6. Banegas JR, Rodríguez-Artalejo F, de la Cruz JJ, Guallar-Castillón P, del Rey J. Blood Pressure in Spain. Distribution, Awareness, Control, and Benefits of a Reduction in Average Pressure. Hypertension. 1998; 32: 998-1002.

7. Banegas JR, Rodríguez-Artalejo F, Ruilope LM, Graciani A, Luque M, de la Cruz-Troca JJ, et al. Hypertension magnitude and management in the elderly population of Spain. J Hypertens 2002; 20: 2157-2164.
8. Marín R, de la Sierra A, Armario P, Campo C, Banegas JR, Gorostidi M, en representación de la Sociedad Española de Hipertensión-Liga Española para la Lucha contra la Hipertensión Arterial (SEH-LELHA). Guía sobre el diagnóstico y tratamiento de la hipertensión arterial en España 2005. Med Clin (Barc). 2005; 125: 24-34.

9. Whelton PK. Epidemiology of hypertension. Lancet. 1994; 344: 101-106.

10. Guidelines Committee. 2003 European Society of Hypertension-European Society of Cardiology guidelines for the management of arterial hypertension. J Hypertens. 2003; 21: 1011-1053.

11. Chobanian AV, Bakris GL, Black HR, Cushman WC, Green LA, Izzo JL, and the National High Blood Pressure Education Program Coordinating Committee. The Seventh Report of the Joint National Committee on Prevention, Detection, Evaluation, and Treatment of High Blood Pressure. Hypertension. 2003; 42: 1206-1252.

12. United Nations. World Population Prospects. Disponible en http://esa.un.org/unpp (consultado el 28 de marzo de 2005).

13. O'Brien E, Mee F, Atkins N, Thomas M. Evaluation of three devices for self-measurement of blood pressure according to the revised BHS Protocol: the Omron HEM-705CP, Philips HP5332, and Nissei DS.-175. Blood Press Monit. 1996; 1: 55-61.

14. Hense HW, Koivisto AM, Kuulasmaa K, Zaborskis A, Kupsc W, Tuomilehto J, for the WHO MONICA Project. Assessment of blood pressure measurements quality in the baseline surveys of the WHO MONICA Project. J Hum Hypertens. 1995; 9: 935-946.

15. Grupo Cooperativo del Programa Hipertensión Navarra-82. La hipertensión arterial en Navarra. En: Pardell H, director. La hipertensión arterial en España. Madrid: Liga Española para la Lucha contra la Hipertensión Arterial; 1984. p. 109-18.

16. Ramírez P, Moreno S, Abellán J, Merino E, Merino J. Prevalencia de la hipertensión arterial y su distribución en la población murciana. Med Clin (Barc) 1984; 83: 188-91.

17. Aranda P, Villar J, y el Grupo colaborativo andaluz sobre factores de riesgo vascular. Estudio epidemiológico andaluz sobre factores de riesgo vascular. Estudio Al Andalus 1990. Sevilla: Consejería de Salud; 1993.

18. Compañ L, Vioque J, Quiles J, Hernández-Aguado I, Borrás F. Prevalencia y control de la hiper- 
tensión arterial en la población adulta de la Comunidad Valenciana, 1994. Med Clin (Barc) 1998; 110: 328-33.

19. Puras A, Sanchis C, Artigao LM, Divison JA. Prevalence, awareness, treatment, and control of hypertension in a Spanish population. Eur J Epidemiol 1998; 14: 31-6.

20. Motero J, Aranda P, Atienza F, Márquez F, Quesada MC, en nombre del Grupo Onuba-89. Epidemiología de la hipertensión arterial en la población adulta de la provincia de Huelva. Hipertensión 1992; 9: 278-87.

21. Pascual S, Carrasco ML, Vázquez A, Abrigar P, Santos J, Torres G, et al. Estudio epidemiológico de la hipertensión arterial y su relación con otros factores de riesgo cardiovascular en la ciudad de Burgos. Hipertensión 1991; 8: 364-69.

22. Pérez C, Gil VF, Pascual R, Aparicio JM, Belda J, Rubio C, et al. Estudio epidemiológico de hipertensión arterial en la provincia de Alicante. Hipertensión 1993; 10: 118-23.
23. Abelleira A, Otero A, Raposo C. Factores de riesgo cardiovascular en la población adulta de la provincia de Orense. Estudio epidemiológico. Hipertensión 1994; 11: 138-53.

24. Medrano MJ, Cerrato E, Boix R, Delgado-Rodríguez M. Factores de riesgo cardiovascular en la población española: metaanálisis de estudios transversales. Med Clin (Barc) 2005; 124: 606-12.

25. Wang Y, Wang QJ. The prevalence of prehypertension and hypertension among US adults according to the new Joint National Committee Guidelines. New challenges of the old problem. Arch Intern Med 2004; 164: 2126-34.

26. Ruiz LM. Resultados de Cantabria. En: Ruiz Ceballos LM, director. Análisis de situación de la hipertensión arterial en Cantabria. Santander: Consejería de Sanidad y Bienestar Social. Diputación Regional de Cantabria; 1989. p. 259-67.

27. Plan Integral de Cardiopatía Isquémica 20042007. Madrid: Ministerio de Sanidad y Consumo; 2003. 